ARTICLE

https://doi.org/10.1038/s41467-019-13509-3

OPEN

\title{
Solid-to-liquid phase transitions of sub-nanometer clusters enhance chemical transformation
}

Juan-Juan $\operatorname{Sun}^{1} \&$ Jun Cheng (1) ${ }^{1 \star}$

Understanding the nature of active sites is crucial in heterogeneous catalysis, and dynamic changes of catalyst structures during reaction turnover have brought into focus the dynamic nature of active sites. However, much less is known on how the structural dynamics couples with elementary reactions. Here we report an anomalous decrease in reaction free energies and barriers on dynamical sub-nanometer Au clusters. We calculate temperature dependence of free energies using ab initio molecular dynamics, and find significant entropic effects due to solid-to-liquid phase transitions of the Au clusters induced by adsorption of different states along the reaction coordinate. This finding demonstrates that catalyst dynamics can play an important role in catalyst activity.

\footnotetext{
${ }^{1}$ Collaborative Innovation Center of Chemistry for Energy Materials (iChEM), State Key Laboratory of Physical Chemistry of Solid Surfaces, College of 
T he concept of active site in heterogeneous catalysis was coined by Taylor ${ }^{1}$ nearly a century ago, and ever since chemists have made great endeavors to characterize the structures of the active sites of catalysts and elucidate how the special arrangements of atoms accelerate chemical reactions ${ }^{2,3}$. The rationale behind is that molecular understanding of the active sites would help design and search for better catalysts ${ }^{4}$. A prominent example is the Au catalyst ${ }^{5,6}$; it had been long thought of as an inert material incapable of catalyzing any reactions ${ }^{7}$ until Haruta et al. discovered that it becomes active when being made into nanoparticles ${ }^{6,8}$. It is now well established that the stepped or kink sites abundant in nanoparticles consist of under-coordinated $\mathrm{Au}$ atoms that are active in breaking chemical bonds.

The notion of sites however often gives a somewhat static or rigid picture of the active sites of catalysts with fixed atomic configurations. Although in recent years the advent of in situ spectroscopic and microscopic techniques ${ }^{9-13}$ and electronic structure calculation methods has allowed for investigating the dynamic evolution of the structures of catalysts under reaction conditions ${ }^{14-19}$, the static perspective has been still taken in identifying the active sites under environmental conditions and monitoring the transformation of one type of active sites into another. For example, ab initio molecular dynamics (AIMD) simulations have been used to explore the diversity of structural configurations of clusters, and however elementary reactions have been still calculated on given cluster structures using static optimization techniques at $0 \mathrm{~K}^{15}$. This is justifiable because from the viewpoint of elementary reactions, which occur at the time scale of picoseconds, the catalytic sites are essentially static considering the catalyst structures usually evolve at macroscopic timescales $^{10,12,20}$. The question arises, what if the time scale of the dynamic evolution of catalyst structures overlaps with that of chemical reactions? In the following, we will show that this is indeed the case for the reactions occurring on sub-nanometer clusters, and moreover we find an anomalous decrease in the reaction free energies and barriers owing to entropic effects that are attributable to (quasi-)first-order solid-to-liquid phase transitions of catalyst clusters during the course of the chemical reactions. This discovery provides a new insight into our understanding of catalysis on small clusters widely existing in supported catalysts.

\section{Results}

Free energies of $\mathbf{O}_{2}$ dissociation on $\mathbf{A u}_{13}$. Here, we choose $\mathrm{O}_{2}$ activation on small Au clusters as example, because it is a key step in the first reaction (i.e. $\mathrm{CO}$ oxidation) demonstrated for the $\mathrm{Au}$ catalysis by Haruta et al. ${ }^{8}$ and numerous other oxidation reaction ${ }^{21}$ such as alkene oxidation ${ }^{22,23}$. We employ AIMD simulation ${ }^{24,25}$ which is very suitable for the purpose of both accounting for the chemisorption of molecules on clusters and the dynamic evolution of clusters when breaking chemical bonds. We first calculate the free energy profiles of $\mathrm{O}_{2}$ dissociation reaction on $\mathrm{Au}_{13}$ cluster (see Fig. 1a, Supplementary Fig. 1a). The free energy profiles are obtained by computing the potential of mean force (PMF) along the reaction coordinate of O-O distance. It is shown that the temperature plays an important role in the structure dynamics of clusters ${ }^{26-28}$, and therefore we investigate the dynamic catalysis on the cluster at a temperature range varying from $120 \mathrm{~K}$ to $600 \mathrm{~K}$. We also calculate the reaction at $0 \mathrm{~K}$ using static geometry optimization for comparison. As shown in Supplementary Fig. 2, the cluster structure undergoes significant change along the reaction path, more so at higher temperatures, in contrast that it hardly changes at $0 \mathrm{~K}$.

The calculated free energy profiles and the temperature dependence of reaction free energies and barriers are shown in
Fig. 1b, c, and the corresponding PMF data is given in Supplementary Fig. 3. The hysteresis (Supplementary Fig. 3d) is very small in thermodynamic integration and the statistical errors in the PMF (Supplementary Fig. 3e, f) are negligible. It is evident that with the temperature increasing, both the reaction free energy and barrier decrease dramatically (Fig. 1c, Supplementary Table 1 for detailed values). In contrast, such temperature dependence of free energies is not observed by the commonly used geometry optimization method (the hollow circle of Fig. 1c, Supplementary Table 2 for detailed values). The static calculation corrects for entropic terms based on models such as harmonic oscillator, which are largely canceled in energy differences, and hence misses configurational entropies owing to dynamic fluctuation of the cluster structure. In the PMF calculation, AIMD samples the ensemble of the relevant configurations of the cluster consisting of both the adsorbed $\mathrm{O}_{2}$ and the $\mathrm{Au}_{13}$ cluster, and therefore takes all entropic contributions into consideration. The effect of structural dynamics is also manifested by the finding that the $\mathrm{O}-\mathrm{O}$ distance at the transition state (TS) of the reaction free energy pathway decreases from 2.2 to $1.8 \AA$ when the temperature increases from 120 to $600 \mathrm{~K}$, in comparison to the value of $2.1 \AA$ obtained from the static calculation (Supplementary Fig. 4).

It is interesting to note from Fig. $1 \mathrm{c}$ that the curves of the free energy and barrier against the temperature show three characteristic regions, with a steep transition region at the temperature range of about $300-400 \mathrm{~K}$ separating the low and high temperature regions with small slopes. This behavior is even clearer if taking the derivatives of the free energies with respect to the temperature, which gives the entropy changes as a function of the temperature. As illustrated in Fig. 1d, the entropy changes show Gaussian-like distributions peaked in the temperature range of free energy transition. The peak heights are surprisingly large, on the order of about $1800 \mathrm{~J} \mathrm{~mol}^{-1} \mathrm{~K}^{-1}$ and $600 \mathrm{~J} \mathrm{~mol}^{-1} \mathrm{~K}^{-1}$ (i.e., for one mole of the cluster) at $\sim 340 \mathrm{~K}$ for the reaction entropy change $\left(\Delta_{\mathrm{r}} S\right)$ and activation entropy $\left(\Delta S^{\ddagger}\right)$, respectively. It is these large entropy changes that give rise to the drastic decreases in free energies at the transition temperature range.

Anomalous reaction entropies and phase transitions of $\mathrm{Au}_{13}$. What causes the temperature dependent behavior of the reaction free energies on the cluster and what is the physical origin behind the enormous entropy changes at the transition region? It is conceivable that such large entropy changes often occur in phase transitions ${ }^{29}$. We thus explore how the total energies change with varying temperature, and calculate the canonical caloric curves $\langle\mathrm{E}\rangle(\mathrm{T})$ of the reactant, TS and product, as shown in Fig. 2a-c (also see Supplementary Fig. 5 for the convergence of time averages of total energies). All three caloric curves show a similar trend that the total energies linearly increase with increasing temperature at low and high temperature ranges, with sudden jumps in between as indicated with gray areas in Fig. 2a-c. This is indeed characteristic of phase transitions, and consistent with the common view that small finite-size systems like clusters can show quasi-first-order phase transitions, in which there is no welldefined transition (melting) temperature but rather a range of temperature where both phases coexist ${ }^{30}$. What is intriguing is that the three curves have different transition temperature ranges, which can be clearly revealed from the specific heat curves $\mathrm{Cv}(\mathrm{T})$ by taking the derivatives of the caloric curves $\langle\mathrm{E}\rangle(\mathrm{T})$ against the temperature. As illustrated in Fig. 2e-g, unlike the bulk limit of a first-order phase transition in which the specific heat is infinite and discontinuous at the melting point, the specific heat curves $\mathrm{Cv}(\mathrm{T})$ are continuous and have peaks with finite widths. For convenience, we define the temperature at the peak maximum as 
a

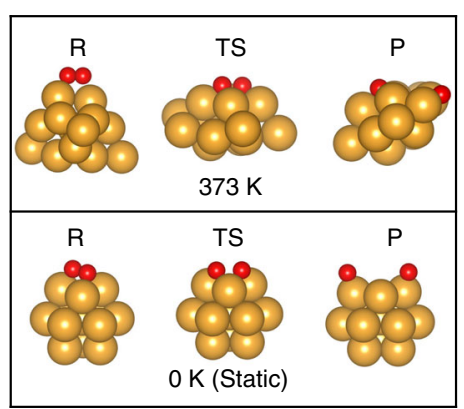

c

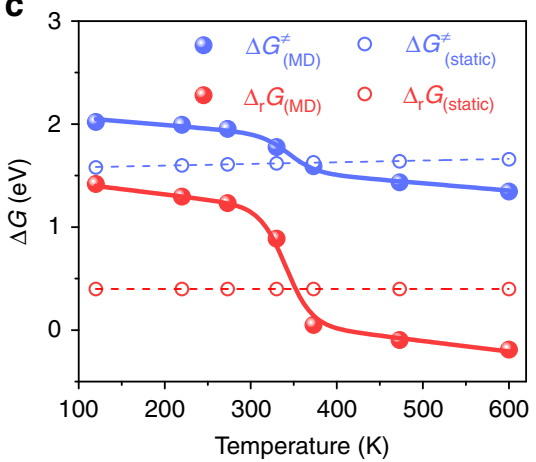

b

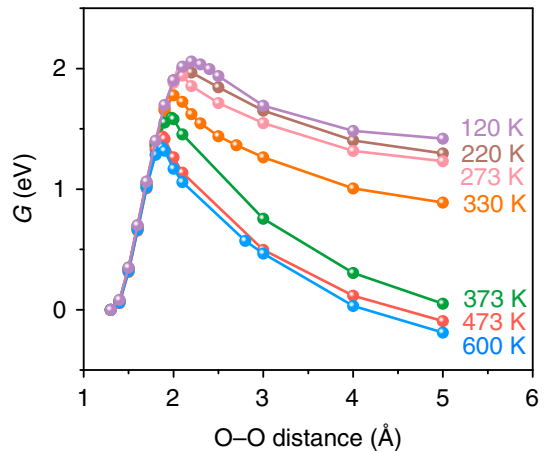

d

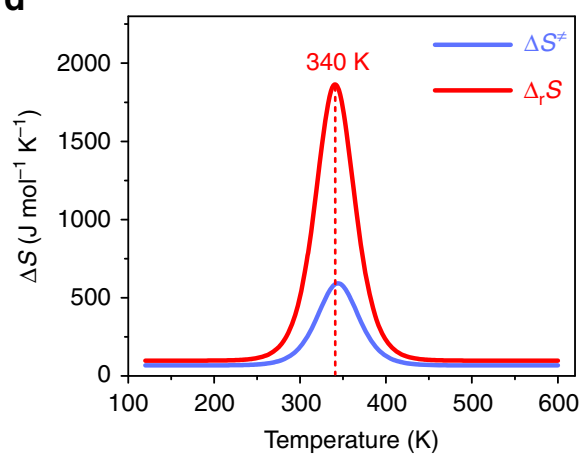

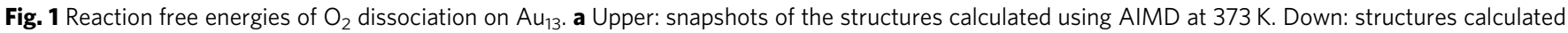

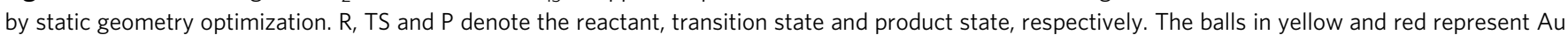
and $\mathrm{O}$ atoms, respectively. b The free energy profiles of $\mathrm{O}_{2}$ dissociation on $\mathrm{Au}_{13}$ under different temperature calculated using $\mathrm{AIMD}$. c Temperature dependence of reaction free energies $\left(\Delta_{\mathrm{r}} \mathrm{G}\right.$, red) and free energy barriers ( $\Delta \mathrm{G}$, blue). Free energies are referenced to those of the reactant states. The filled and hollow circles represent the calculated energies using AIMD and static geometry optimization method, respectively, and the solid and dash curves are

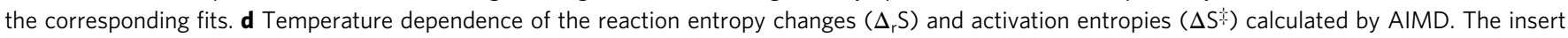
value indicates the temperature of maximum entropy change.

the melting temperature in the quasi-first-order phase transition. Thus, the melting temperature for the reactant state is about $390 \mathrm{~K}$, similar to that of bare $\mathrm{Au}_{13}(\sim 413 \mathrm{~K})^{31}$, higher than that of the product state $(\sim 340 \mathrm{~K})$, and the melting temperature of TS is in between (i.e., $\sim 350 \mathrm{~K}$ ).

These differences in the melting temperatures for different states along the reaction coordinate can have great impact on the entropy and free energy changes. Comparing the phase changes of the reactant and product, at the temperature below $\sim 290 \mathrm{~K}$ both the reactant and product states are solid, leading to a minor $\Delta_{\mathrm{r}} \mathrm{S}$ (the white area in Fig. 2d). When increasing the temperature to the range $290-340 \mathrm{~K}$, the product becomes the solid-liquid coexistence state (the orange area in Fig. 2e), with dramatic entropy increase of the product, while the reactant remains in the solid state (the white area in Fig. $2 \mathrm{f}$ ). Consequently, $\Delta_{\mathrm{r}} \mathrm{S}$ will increase (the orange area in Fig. 2d), to the same extent as the entropy increase in the phase transition of the product. When the temperature increases to $340 \mathrm{~K}$, the reactant starts to melt, reaching the solid-liquid coexistence state (the green area in Fig. 2f). The entropy increase of the reactant thus compensates that of the product, leading to reduction in $\Delta_{\mathrm{r}} \mathrm{S}$ (the green area in Fig. 2d). When the temperature is higher than $420 \mathrm{~K}$, both the reactant and product completely transform to the liquid states, resulting in a small $\Delta_{\mathrm{r}} \mathrm{S}$ (the white area in Fig. $2 \mathrm{~d}$ ). Similarly, the temperature dependence of the activation entropy $\Delta S^{\ddagger}$ (shown in Fig. $2 \mathrm{~h}$ ) can also be explained with the different phase transition behaviors of the reactant (shown in Fig. 2f) and TS (shown in Fig. 2g).

The underlying mechanism of the anomalous entropy change is best illustrated in the Fig. $2 \mathrm{i}$. The small-size cluster is subjected to the strong influence of the adsorption of reaction species. The change of the state of the adsorbate (e.g., $\mathrm{O}_{2}$ molecule vs two $\mathrm{O}$ atoms) could even alter the phase transition temperature of the cluster. Discrepancy between the melting temperatures of the cluster with different states of the species will inevitably result in a transition temperature range in which one state melts while the other doesn't, and hence the anomalous increase in the reaction entropy. Thus, it is possible to search for the favorable temperature range in which the cluster undergoes a solid-toliquid phase transition along the reaction coordinate, lowering the reaction free energy and barrier.

$\mathrm{O}_{2}$ dissociation on $\mathrm{Au}_{8} / \mathrm{MgO}$ and $\mathrm{H}_{2} \mathrm{O}$ dissociation on $\mathrm{Au}_{13}$. In heterogeneous catalysis, many active catalysts are metal clusters dispersed on some supports. We therefore further study $\mathrm{O}_{2}$ dissociation on the $\mathrm{Au}_{8} / \mathrm{MgO}$ model ${ }^{32}$ using AIMD. Similar to $\mathrm{Au}_{13}$, it is found that the structure of $\mathrm{Au}_{8}$ supported on $\mathrm{MgO}$ calculated by AIMD at finite temperatures is rather dynamical, and different from that obtained by static geometry optimization (Fig. 3a and Supplementary Fig. 1c). With the increase of temperature, both the reaction free energy and barrier decrease (Supplementary Fig. $6 \mathrm{~b}, \mathrm{c}$ ). In particular, the temperature dependence of $\Delta_{\mathrm{r}} \mathrm{S}$ on $\mathrm{Au}_{8} / \mathrm{MgO}$ (Fig. 3b) is very similar to that on $\mathrm{Au}_{13}$ (Fig. 1d), showing a Gaussian-like distribution with a maximum of $\sim 700 \mathrm{~J}$ $\mathrm{mol}^{-1} \mathrm{~K}^{-1}$ around $390 \mathrm{~K}$. This Gaussian peak in entropy again results from the different phase transition temperatures of the reactant and product (Fig. 3b-d, Supplementary Figs. 7 and 8) on the supported cluster, suggesting that this anomalous increase in reaction entropy could also occur on real catalysts. For activation entropy $\Delta \mathrm{S}^{\ddagger}$ on $\mathrm{Au}_{8} / \mathrm{MgO}$, the peak is somewhat absent, the free 

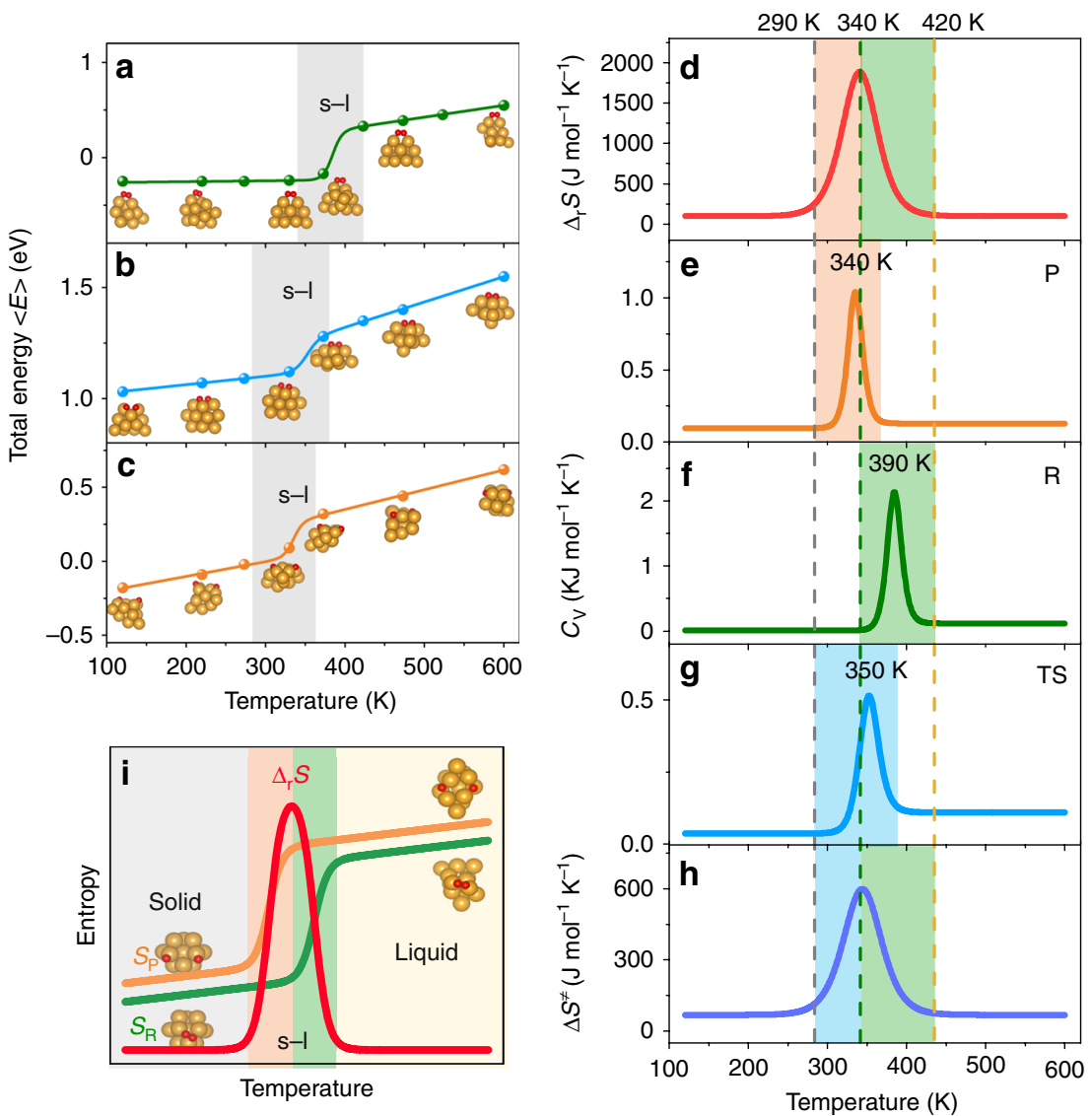

Fig. 2 Correlation between phase transitions of the clusters and entropy changes. a-c Calculated caloric curves $\langle E\rangle(T)$ of the reactant, transition state and product state, respectively. The dots show the calculated values and the lines are fitting curves. The s-l indicates solid-liquid coexistence state. The insets show snapshots of the structures at different temperatures. The balls in yellow and red represent $A u$ and $O$ atoms, respectively. $\mathbf{d}$ Temperature dependence of the reaction entropy change $\left(\Delta_{\mathrm{r}} \mathrm{S}\right)$. e- $\mathbf{g}$ The $C_{v}(T)$ curve of the product $(P)$, reactant $(R)$ and transition state (TS), respectively. The melting temperatures at the peaks are indicated as the inserted values. $\mathbf{h}$ Temperature dependence of activation entropy $(\Delta S)$. $\mathbf{i}$ Schematic illustration of the anomalous reaction entropy change due to the difference in the transition temperature of the solid-to-liquid phase transition of the cluster at the reactant and product state. The curves in red, green and orange indicate the reaction entropy change $\left(\Delta_{\mathrm{r}} \mathrm{S}\right)$, the entropy of the reactant $\left(\mathrm{S}_{R}\right)$ and the entropy of the product $\left(S_{P}\right)$, respectively.

energy barrier decreases almost linearly with temperature (Supplementary Fig. 6c) with a nearly constant activation entropy $\Delta S^{\ddagger}$ of $\sim 60 \mathrm{~J} \mathrm{~mol}^{-1} \mathrm{~K}^{-1}$ (Fig. 3e). This can be attributable to the similar phase transition temperatures of the reactant and TS, as shown in Fig. 3e-g.

Whether the anomalous entropy change is present (i.e., $\Delta_{\mathrm{r}} \mathrm{S}$ vs $\Delta S^{\ddagger}$ on $\mathrm{Au}_{8} / \mathrm{MgO}$ ), or how large is the entropy change (i.e., $\Delta_{\mathrm{r}} \mathrm{S}$ on $\mathrm{Au}_{13} \mathrm{vs}^{\mathrm{Au}_{8}} / \mathrm{MgO}$ ) must depend on the extent of the change of the state of the adsorbed species, and the susceptibility of the cluster to this change. This could explain why the peaks of $\Delta_{\mathrm{r}} \mathrm{S}$ are higher than $\Delta S^{\ddagger}$, since the structure of the TS is more similar to that of the reactant than that of the product is. This would also suggest that different types of reactions may show different behaviors. We thus further calculate $\mathrm{H}_{2} \mathrm{O}$ dissociation reaction on $\mathrm{Au}_{13}$ cluster (Fig. 3h). It is found that the general trends of the reaction free energy and barrier (Supplementary Fig. 9b, Fig. 3i) are indeed very similar to those of $\mathrm{O}_{2}$ dissociation (Fig. 1b, c). The entropy changes $\left(\Delta_{\mathrm{r}} \mathrm{S}\right.$ and $\left.\Delta \mathrm{S}^{\ddagger}\right)$ of $\mathrm{H}_{2} \mathrm{O}$ dissociation show gaussian-like distributions but with smaller peak heights than those of $\mathrm{O}_{2}$ dissociation (Fig. $3 \mathrm{j}$ and Fig. 1d). The Gaussian peak in entropy results from the different phase transitions of the reactant, TS and product as well (Supplementary Figs. 10 and 11). Comparing the adsorption of $\mathrm{H}_{2} \mathrm{O}$ and $\mathrm{O}_{2}$ (Supplementary Fig. 1a, b), it is clear that $\mathrm{O}_{2}$ binds to the cluster stronger than $\mathrm{H}_{2} \mathrm{O}$, indicating stronger influence on the cluster. Perhaps more importantly, after dissociation $\mathrm{O}_{2}$ breaks into two $\mathrm{O}$ atoms each having two bond valences to bind the cluster, rendering even greater impact on the cluster. While, $\mathrm{H}_{2} \mathrm{O}$ breaks into $\mathrm{OH}$ and $\mathrm{H}$ both having single bond valence that can only cast limited impact. Therefore, it is anticipated that not only can the adsorptioninduced phase transitions of small clusters improve the activity at certain temperatures but also can shift the selectivity towards certain types of reactions.

\section{Discussion}

This theoretically discovered phase transition effect may have either been present in some experimental work without being realized or not been observed yet since one has to closely look into the interplay between cluster dynamics and temperature. Real catalysts usually have an ensemble of particles with a distribution of sizes, in which small clusters have less isomers ${ }^{33}$ and large particles tend to be more rigid ${ }^{34}$, both resulting in small entropic effects. Thus, we expect that the dynamic effect will be most pronounced for clusters consisting of a few tens of atoms. Considering that the activity of catalysts results from collective contributions of all clusters with different sizes, there may exist some difficulties in identifying this phase transition effect experimentally. Interestingly, it has been reported that the most active $\mathrm{Au}$ clusters for $\mathrm{CO}$ oxidation consist of tens of $\mathrm{Au}$ atoms ${ }^{35}$ This size-dependent activity of Au clusters may be related to the 


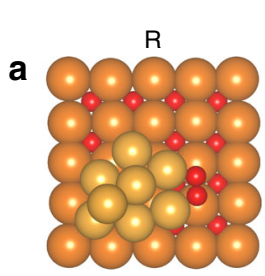

TS

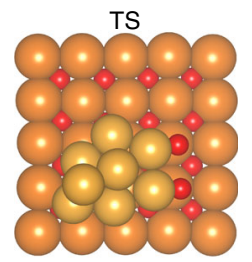

$\mathrm{P}$

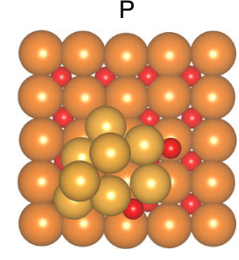

h

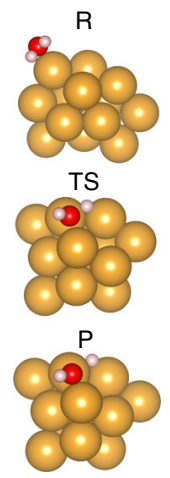

$290 \mathrm{~K} 390 \mathrm{~K} 500 \mathrm{~K}$

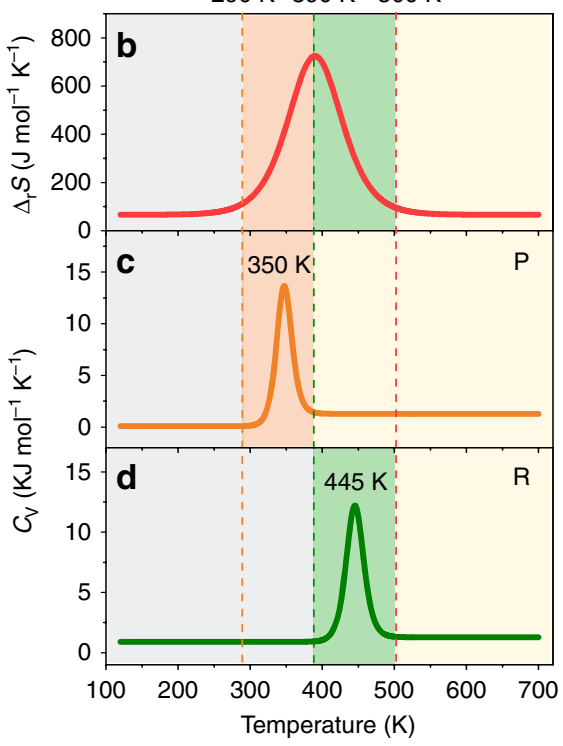

Solid

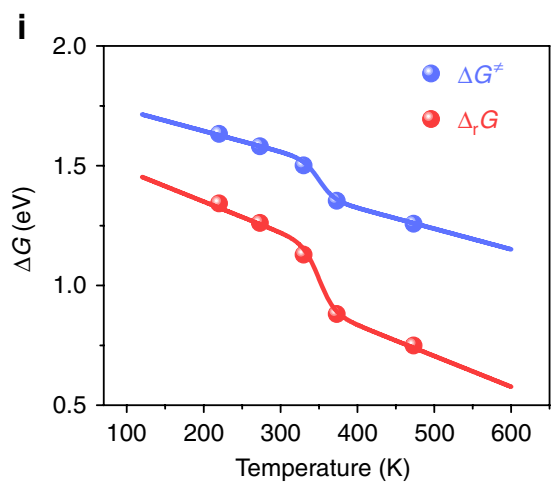

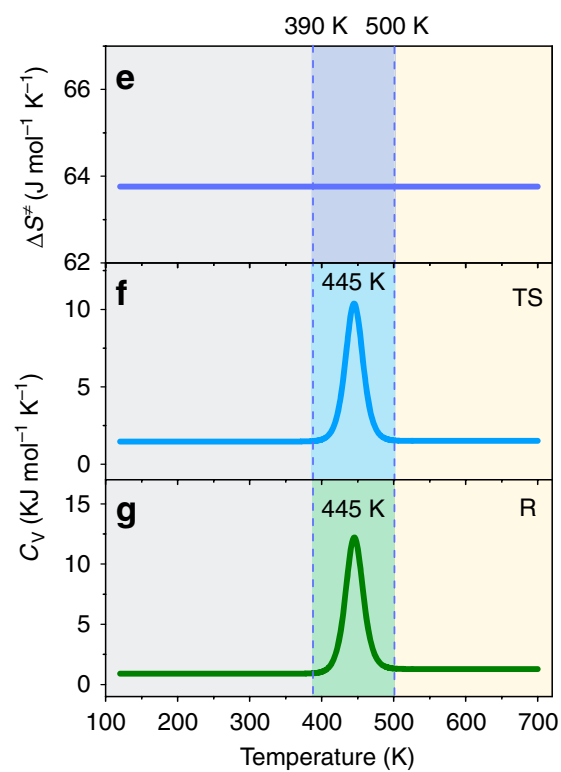

TS coexist

P coexist

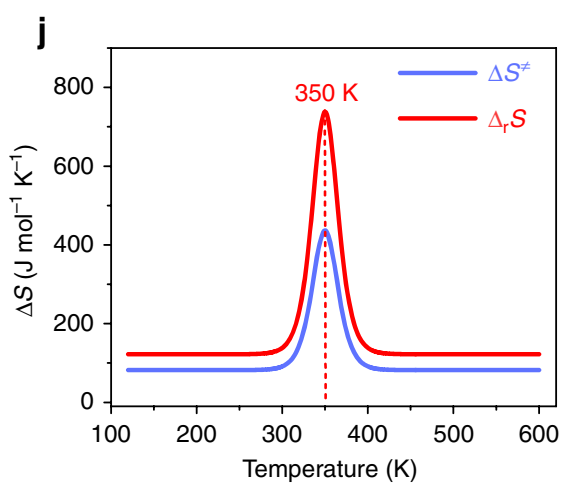

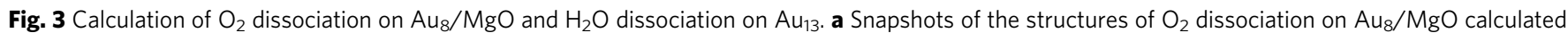

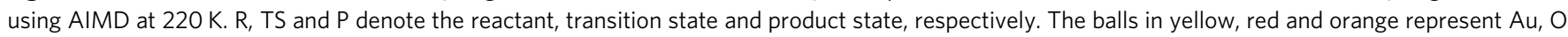
and $\mathrm{Mg}$ atoms, respectively. b Temperature dependence of the reaction entropy change $\left(\Delta_{\mathrm{r}} \mathrm{S}\right)$. $\mathbf{c}, \mathbf{d}$ The $\mathrm{C}_{\mathrm{v}}(\mathrm{T})$ curve of the product and reactant, respectively. e Temperature dependence of the activation entropy $(\Delta \mathrm{S})$. $\mathbf{f}, \mathbf{g}$ The $\mathrm{C}_{\mathrm{v}}(\mathrm{T})$ curve of the TS and reactant, respectively. The melting temperatures at the peaks are indicated as the inserted values. $\mathbf{h}$ Snapshots of the structures of the reactant, TS and product of $\mathrm{H}_{2} \mathrm{O}$ dissociation on Au $\mathbf{u}_{13}$ calculated using AIMD at $220 \mathrm{~K}$. The balls in yellow, red and white represent $\mathrm{Au}, \mathrm{O}$ and $\mathrm{H}$ atoms, respectively. $\mathbf{i}$ Temperature dependence of the reaction free energy $\left(\Delta_{\mathrm{r}} \mathrm{G}\right)$ and free energy barrier $(\Delta \mathrm{G})$. The dots show the calculated values and the lines are fitting curves. $\mathbf{j}$ Temperature dependence of the reaction entropy change $\left(\Delta_{\mathrm{r}} \mathrm{S}\right)$ and activation entropy $(\Delta \mathrm{S})$. The insert value indicates the temperature of maximum entropy change.

dynamic effect. Furthermore, supports can significantly influence cluster dynamics. Comparing the results above between $\mathrm{Au}_{13}$ and $\mathrm{Au}_{8} / \mathrm{MgO}$, it can be seen that $\mathrm{MgO}$ can reduce cluster dynamics to some extent and thus the entropic effect induced by phase transition.

However, we envision that with the development of synthesis methods to obtain more uniform sized cluster catalysts and in situ atomic characterization techniques, this phase transition effect could be realized by experiment by investigating the temperature effect on catalyst dynamics and activity. It would also offer possible explanations for experimental observations on the effects of cluster size $\mathrm{e}^{36,37}$, types of supports ${ }^{38}$, co-adsorption ${ }^{39}$, etc. Understanding these effects on catalyst dynamics will certainly merits future studies.

Finally, we'd like to draw a parallel with enzyme catalysis. First proposed by Linus Pauling ${ }^{40}$, enormous acceleration of reaction rates is achieved by enzymes through an enhanced TS stabilization mechanism. This concept has played a key role in drug design for decades, guiding development of catalytic antibodies that are small, stable molecules mimicking the structures of activated TS complexes in enzymes. However, these antibodies often lead to minor rate enhancements. It is now believed that there was too much focus on rigid TS structures, and protein dynamics (motion) has strong influence on the catalytic activity of enzymes. The latter is becoming the central issue in enzyme catalysis, although its role is still under debate ${ }^{41}$. In analogy with protein dynamics, we show that catalyst dynamics can significantly improve the activity of sub-nanometer clusters through a solid-to-liquid phase transition mechanism, which in turn may shed some light on enzyme catalysis.

To summarize, we study the structural dynamics of small Au clusters and its effects on temperature dependence of free energies of model reactions using AIMD, and discover the abnormal changes of free energies and entropies at certain temperature ranges. We, for the first time, show solid-to-liquid phase transitions of the clusters induced by adsorption of reaction species can facilitate the reactions. Our work highlights the importance of catalyst dynamics in understanding catalytic activity in 
heterogeneous catalysis, and opens up promising ways for optimizing the activity and selectivity of catalysts.

\section{Methods}

Computational models. The structures of bare $\mathrm{Au}_{13}$, and $\mathrm{Au}_{8}$ supported on clean $\mathrm{MgO}(001)$ were constructed to study the structural dynamics of the clusters and its effects on catalysis. The $\mathrm{Au}_{13}$ cluster was initially built with a highly symmetric cuboctahedral $\left(\mathrm{O}_{h}\right)$ structure. $\mathrm{O}_{2}$ prefers to adsorb on the hollow site (Supplementary Fig. 1a), and $\mathrm{H}_{2} \mathrm{O}$ prefers to adsorb on the top site (Supplementary Fig. 1b). The $\mathrm{Au}_{13}$ cluster was simulated in a cubic cell of $15 \times 15 \times 15 \AA^{3}$. A fourlayer $\mathrm{MgO}(001)-\mathrm{p}(4 \times 4)$ slab was used for the support of the $\mathrm{Au}_{8}$ cluster. The cells were modeled under $3 \mathrm{D}$ periodic boundary conditions, and the slabs and their images were separated by vacuum with a length of $15 \AA . \mathrm{O}_{2}$ molecule prefers to adsorb at the interface between the cluster and the support (Supplementary Fig. 1c).

Density functional theory (DFT) calculation. The AIMD simulations were carried out using the freely available program package CP2K/Quickstep ${ }^{42}$ The DFT implementation is based on a hybrid Gaussian plane wave (GPW) scheme, the orbitals are described by an atom centered Gaussian-type basis set, and an auxiliary plane wave basis set is used to re-expand the electron density in the reciprocal space. Perdew-Burke-Ernzerhof (PBE) functional ${ }^{43}$ with Grimme's dispersion correction ${ }^{44}$ was used. The core electrons were represented by analytic GoedeckerTeter-Hutter (GTH) pseudopotentials $s^{45,46}$. For valence electrons $\left(5 \mathrm{~d}^{10} 6 \mathrm{~s}^{1}\right.$ for $\mathrm{Au}$, $2 s^{2} 2 p^{4}$ for $\mathrm{O}, 2 s^{2} 2 p^{6} 3 s^{2}$ for $M g, 1 s^{1}$ for $H$ ), the Gaussian basis sets were double- $\zeta$ basis functions with one set of polarization functions (DZVP) ${ }^{47}$. We performed the spin-polarized DFT calculations on $\mathrm{Au}_{13}$ and $\mathrm{Au}_{8} / \mathrm{MgO}$. The spin state of $\mathrm{O}_{2}$ on $\mathrm{Au}_{13}$ and $\mathrm{Au}_{8} / \mathrm{MgO}$ is doublet and singlet, respectively, and the spin state of $\mathrm{H}_{2} \mathrm{O}$ on $\mathrm{Au}_{13}$ is doublet.

Free energy calculation. In the work, we have calculated the reaction free energy profiles by combining AIMD, constrained MD and thermodynamic integration. The reaction free energies (barriers) can be obtained by integrating potentials of mean force (PMF) over a chosen reaction coordinate ${ }^{48}$, i.e., the $\mathrm{O}-\mathrm{O}$ distance of $\mathrm{O}_{2}$ dissociation reaction and the $\mathrm{H}-\mathrm{O}$ distance for $\mathrm{H}_{2} \mathrm{O}$ dissociation. The PMF is calculated using a Lagrange multiplier method, by averaging the force applied on the system to keep the reaction coordinate constant in AIMD runs ${ }^{49}$. In the AIMD simulations, canonical ensemble (NVT) conditions were imposed by a NoseHoover thermostat under various temperatures. The MD time step is set to $0.5 \mathrm{fs}$. In AIMD runs, the trajectories of the first 5-10 ps were regarded as equilibrium periods to ensure equilibria of the systems, followed by another 5-15 ps of production periods for data analysis. For the size of systems studied in this work, the time scale of $\sim 10$ ps is sufficient to obtain well converged PMFs. as evident by the time accumulating averages shown in Supplementary Fig. 3a, b. Integrating the forces against the distance gives the free energy profile of the $\mathrm{O}_{2}$ dissociation reaction (Supplementary Fig. 3c). In the force-distance curves, three points crossing the force zero correspond to the reactant, transition and product state, respectively. Note that the hysteresis in our thermodynamic integration is very small. As shown in Supplementary Fig. 3d, when calculating the mean force at the O-O distance of $2 \AA$, we have started the structure models in both forward and backward directions, i.e., the models with O-O distances of $1.9 \AA$ and $2.1 \AA$, respectively. The corresponding averages of the mean forces are very similar, i.e., $-0.113 \mathrm{eV} \AA^{-1}$ for the initial structure with $\mathrm{O}-\mathrm{O}$ distance to be $1.9 \AA$ (blue) and $-0.106 \mathrm{eV} \AA^{-1}$ for O-O distance of $2.1 \AA$ (red), indicating good convergence of our PMF calculations. To calculate the entropy change, we firstly fit the temperature dependent free energy curves, and then the entropy was obtained by taking the temperature derivative. Similarly, the heat capacity was obtained by taking the temperature derivative of the fitted canonical caloric curve $\langle\mathrm{E}\rangle(\mathrm{T})$

For comparison, the Gaussian $09 \operatorname{code}^{50}$ was also used for geometry optimization calculation of $\mathrm{O}_{2}$ dissociation on $\mathrm{Au}_{13}$. The PBE1PBE ${ }^{51}$ functional was employed to optimize the geometries of $\mathrm{Au}-\mathrm{O}_{2}$ complex. The aug-cc-pvtz ${ }^{52}$ and Lanl2 $\mathrm{DZ}^{53}$ basis sets were employed for $\mathrm{O}$ atom and $\mathrm{Au}$ atom, respectively. During the structure optimization, all the atoms ( $\mathrm{Au}$ and $\mathrm{O}$ atoms) were allowed to relax. The vibrational frequency calculations were carried out to identify the stationary points and transition states (TS) with zero and one imaginary frequency, respectively. The intrinsic reaction coordinate (IRC) calculation was also performed to verify that the transition state connects correctly to the expected minima.

\section{Data availability}

The data that support the findings of this study are available from the corresponding author upon reasonable request.

Received: 20 September 2019; Accepted: 5 November 2019; Published online: 27 November 2019

\section{References}

1. Taylor, H. S. A theory of the catalytic surface. Proc. Roy. Soc. A 108, 105-111 (1925).

2. Hansen, T. W. et al. Atomic-resolution in situ transmission electron microscopy of a promoter of a heterogeneous catalyst. Science 294, 1508-1511 (2001).

3. Nørskov, J. K. et al. The nature of the active site in heterogeneous metal catalysis. Chem. Soc. Rev. 37, 2163-2171 (2008).

4. Greeley, J. P. Active site of an industrial catalyst. Science 336, 810-812 (2012)

5. Hutchings, G. J. Vapor phase hydrochlorination of acetylene: correlation of catalytic activity of supported metal chloride catalysts. J. Catal. 96, 292-295 (1985).

6. Haruta, M., Kobayashi, T., Sano, H. \& Yamada, N. Novel gold catalysts for the oxidation of carbon monoxide at a temperature far below $0{ }^{\circ} \mathrm{C}$. Chem. Lett. 16, 405-408 (1987).

7. Hammer, B. \& Nørskov, J. K. Why gold is the noblest of all the metals. Nature 376, 238-240 (1995).

8. Mitsutaka, O., Tadahiro, F., Jiahui, H. \& Tamao, I. A career in catalysis: Masatake Haruta. ACS Catal. 5, 4699-4707 (2015).

9. Hofmann, S. et al. In situ observations of catalyst dynamics during surfacebound carbon nanotube nucleation. Nano. Lett. 7, 602-608 (2007).

10. Vendelbo, S. B. et al. Visualization of oscillatory behaviour of Pt nanoparticles catalysing CO oxidation. Nat. Mater. 13, 884-890 (2014).

11. Branko, Z. et al. Dynamic restructuring drives catalytic activity on nanoporous gold-silver alloy catalysts. Nat. Mater. 16, 558-564 (2016).

12. Kamiuchi, N. et al. Self-activated surface dynamics in gold catalysts under reaction environments. Nat. Commun. 9, 2060 (2018).

13. Derita, L. et al. Structural evolution of atomically dispersed Pt catalysts dictates reactivity. Nat. Mater. 18, 746-751 (2019).

14. Wang, Y. G. et al. The role of reducible oxide-metal cluster charge transfer in catalytic processes: new insights on the catalytic mechanism of $\mathrm{CO}$ oxidation on $\mathrm{Au} / \mathrm{TiO}_{2}$ from ab initio molecular dynamics. J. Am. Chem. Soc. 135, 10673-10683 (2013).

15. Wang, Y. G., Mei, D., Glezakou, V. A., Li, J. \& Rousseau, R. Dynamic formation of single-atom catalytic active sites on ceria-supported gold nanoparticles. Nat. Commun. 6, 6511 (2015).

16. Taccardi, N. et al. Gallium-rich Pd-Ga phases as supported liquid metal catalysts. Nat. Chem. 9, 862-867 (2017)

17. Liu, X., Wen, X. \& Hoffmann, R. Surface activation of transition metal nanoparticles for heterogeneous catalysis: what we can learn from molecular dynamics. ACS Catal. 8, 3365-3375 (2018).

18. Zandkarimi, B. \& Alexandrova, A. N. Dynamics of subnanometer Pt clusters can break the scaling relationships in catalysis. J. Phys. Chem. Lett. 10, 460-467 (2019).

19. Zandkarimi, B. \& Alexandrova, A. N. Surface-supported cluster catalysis: ensembles of metastable states run the show. Wiley Interdiscip. Rev. Comput. Mol. Sci. 9, e1420 (2019).

20. Kalz, F. K. et al. Future challenges in heterogeneous catalysis: understanding catalysts under dynamic reaction conditions. ChemCatChem 9, 17-29 (2017).

21. Montemore, M. M., van Spronsen, M. A., Madix, R. J. \& Friend, C. M. $\mathrm{O}_{2}$ activation by metal surfaces: implications for bonding and reactivity on heterogeneous catalysts. Chem. Rev. 118, 2816-2862 (2018).

22. Turner, M. et al. Selective oxidation with dioxygen by gold nanoparticle catalysts derived from 55-atom clusters. Nature 454, 981-983 (2008).

23. Qian, L. P. et al. Stable and solubilized active Au atom clusters for selective epoxidation of cis-cyclooctene with molecular oxygen. Nat. Commun. 8, 14881 (2017).

24. Car, R. \& Parrinello, M. Unified approach for molecular dynamics and density-functional theory. Phys. Rev. Lett. 55, 2471-2474 (1985).

25. Marx, D. \& Hutter, J. Ab Initio Molecular Dynamics: Basic Theory and Advanced Methods. (Cambridge University Press, 2009).

26. Krishnamurty, S. et al. Ab initio molecular dynamical investigation of the finite temperature behavior of the tetrahedral $\mathrm{Au}_{19}$ and $\mathrm{Au}_{20}$ clusters. J. Phys. Chem. A 111, 10769-10775 (2007).

27. Koga, K., Ikeshoji, T. \& Sugawara, K. I. Size- and temperature-dependent structural transitions in gold nanoparticles. Phys. Rev. Lett. 92, 115507 (2004).

28. Zhai, H. C. \& Alexandrova, A. N. Ensemble-average representation of Pt clusters in conditions of catalysis accessed through GPU accelerated deep neural network fitting global optimization. J. Chem. Theory Comput. 12, 6213-6226 (2016).

29. Berry, R. S. \& Smirnov, B. M. Entropy behavior in cluster melting. J. Chem. Phys. 130, 064302 (2009).

30. Valsson, O. \& Parrinello, M. Thermodynamical description of a quasi-firstorder phase transition from the well-tempered ensemble. J. Chem. Theory Comput. 9, 5267-5276 (2013).

31. Rey, C., Gallego, L. J. \& García-Rodeja, J. Molecular-dynamics study of the binding energy and melting of transition-metal clusters. Phys. Rev. B 48, 8253-8262 (1993). 
32. Yoon, B. et al. Charging effects on bonding and catalyzed oxidation of $\mathrm{CO}$ on $\mathrm{Au}_{8}$ clusters on MgO. Science 307, 403-407 (2005).

33. Arslan, H. \& Güven, M. H. Melting dynamics and isomer distributions of small metal clusters. N. J. Phys. 7, 60 (2005).

34. Yang, H. et al. Size-dependent dynamic structures of supported gold nanoparticles in CO oxidation reaction condition. Proc. Natl Acad. Sci. 115, 7700-7705 (2018).

35. Herzing, A. A., Kiely, C. J., Carley, A. F., Landon, P. \& Hutchings, G. J. Identification of active gold nanoclusters on iron oxide supports for CO oxidation. Science 321, 1331-1335 (2008).

36. Sanchez, A. et al. When gold is not noble: nanoscale gold catalysts. J. Phys. Chem. A 103, 9573-9578 (1999).

37. Liu, Y., Tsunoyama, H., Akita, T., Xie, S. \& Tsukuda, T. Aerobic oxidation of cyclohexane catalyzed by size-controlled Au. ACS Catal. 1, 2-6 (2011).

38. Haruta, M. et al. Low-temperature oxidation of $\mathrm{CO}$ over gold supported on $\mathrm{TiO}_{2}, \alpha-\mathrm{Fe}_{2} \mathrm{O}_{3}$, and $\mathrm{Co}_{3} \mathrm{O}_{4}$. J. Catal. 144, 175-192 (1993).

39. Green, I. X., Tang, W., Neurock, M. \& Yates, J. T. Spectroscopic observation of dual catalytic sites during oxidation of $\mathrm{CO}$ on a $\mathrm{Au} / \mathrm{TiO}_{2}$ catalyst. Science 333, 736-739 (2011).

40. Pauling, L. Molecular architecture and biological reactions. Chem. Eng. N. 24, 1375-1377 (1946).

41. Klinman, J. P. \& Kohen, A. Hydrogen tunneling links protein dynamics to enzyme catalysis. Annu. Rev. Biochem. 82, 471-496 (2013).

42. VandeVondele, J. et al. Quickstep: Fast and accurate density functional calculations using a mixed Gaussian and plane waves approach. Comput. Phys. Commun. 167, 103-128 (2005).

43. Perdew, J. P., Burke, K. \& Ernzerhof, M. Generalized gradient approximation made simple. Phys. Rev. Lett. 77, 3865-3868 (1996).

44. Grimme, S., Antony, J., Ehrlich, S. \& Krieg, H. A consistent and accurate ab initio parametrization of density functional dispersion correction (DFT-D) for the 94 elements H-Pu. J. Chem. Phys. 132, 154104 (2010).

45. Goedecker, S., Teter, M. \& Hutter, J. Separable dual-space Gaussian pseudopotentials. Phys. Rev. B 54, 1703-1710 (1996).

46. Hartwigsen, C., Goedecker, S. \& Hutter, J. Relativistic separable dual-space Gaussian pseudopotentials from H to Rn. Phys. Rev. B 58, 3641-3662 (1998).

47. VandeVondele, J. \& Hutter, J. Gaussian basis sets for accurate calculations on molecular systems in gas and condensed phases. J. Chem. Phys. 127, 114105 (2007).

48. Carter, E. A., Ciccotti, G., Carter, E. A., Hynes, J. T. \& Kapral, R. Constrained reaction coordinate dynamics for the simulation of rare events. Chem. Phys. Lett. 156, 472-477 (1989).

49. Sprik, M. \& Ciccotti, G. Free energy from constrained molecular dynamics. J. Chem. Phys. 109, 7737-7744 (1998).

50. Frisch, M. J. et al. Gaussian 09 (Gaussian, Inc., Wallingford, CT, 2009).

51. Ernzerhof, M. \& Scuseria, G. E. Assessment of the Perdew-Burke-Ernzerhof exchange-correlation functional. J. Chem. Phys. 110, 5029-5036 (1999).

52. Dunning, T. H. Gaussian basis sets for use in correlated molecular calculations. I. The atoms boron through neon and hydrogen. J. Chem. Phys. 90, 1007-1023 (1989).
53. Wadt, R. W. \& Hay, P. J. Ab initio effective core potentials for molecular calculations. Potentials for main group elements $\mathrm{Na}$ to Bi. J. Chem. Phys. 82 284-298 (1985)

\section{Acknowledgements}

We thank Y. Gao, H. You and H. Häkkinen for helpful discussion. Funding support was provided by National Natural Science Foundation of China (Grant Nos. 91745103, 2181101075, and 21621091).

\section{Author contributions}

J.C. conceived and designed the project. J.-J.S. performed the calculation. J.C. and J.-J.S. analyzed the results and wrote the manuscript.

\section{Competing interests}

The authors declare no competing interests.

\section{Additional information}

Supplementary information is available for this paper at https://doi.org/10.1038/s41467019-13509-3.

Correspondence and requests for materials should be addressed to J.C.

Peer review information Nature Communications thanks the anonymous reviewer(s) for their contribution to the peer review of this work. Peer reviewer reports are available.

Reprints and permission information is available at http://www.nature.com/reprints

Publisher's note Springer Nature remains neutral with regard to jurisdictional claims in published maps and institutional affiliations.

Open Access This article is licensed under a Creative Commons Attribution 4.0 International License, which permits use, sharing, adaptation, distribution and reproduction in any medium or format, as long as you give appropriate credit to the original author(s) and the source, provide a link to the Creative Commons license, and indicate if changes were made. The images or other third party material in this article are included in the article's Creative Commons license, unless indicated otherwise in a credit line to the material. If material is not included in the article's Creative Commons license and your intended use is not permitted by statutory regulation or exceeds the permitted use, you will need to obtain permission directly from the copyright holder. To view a copy of this license, visit http://creativecommons.org/ licenses/by/4.0/.

(C) The Author(s) 2019 